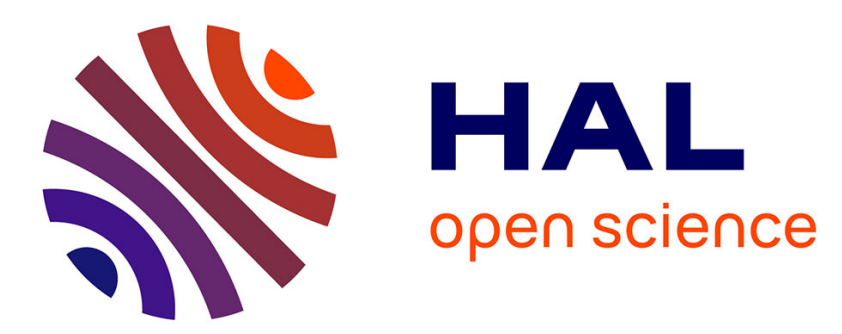

\title{
Evolution representation with limited hosting capacities: A comparison between MSD and IEED
}

\author{
Andres Ovalle, Ahmad Hably, Seddik Bacha
}

\section{To cite this version:}

Andres Ovalle, Ahmad Hably, Seddik Bacha. Evolution representation with limited hosting capacities: A comparison between MSD and IEED. ICIT 2020 - IEEE International Conference on Industrial Technology, Feb 2020, Buenos Aires, Argentina. 10.1109/ICIT45562.2020.9067274 . hal-02398827

\section{HAL Id: hal-02398827 https://hal.science/hal-02398827}

Submitted on 8 Dec 2019

HAL is a multi-disciplinary open access archive for the deposit and dissemination of scientific research documents, whether they are published or not. The documents may come from teaching and research institutions in France or abroad, or from public or private research centers.
L'archive ouverte pluridisciplinaire HAL, est destinée au dépôt et à la diffusion de documents scientifiques de niveau recherche, publiés ou non, émanant des établissements d'enseignement et de recherche français ou étrangers, des laboratoires publics ou privés. 


\title{
Evolution representation with limited hosting capacities: A comparison between MSD and IEED
}

\author{
Andres Ovalle, Ahmad Hably, Seddik Bacha \\ Univ. Grenoble Alpes, CNRS, Grenoble INP*,GIPSA-Lab, G2elab \\ F-38000 Grenoble, France. \\ *Institute of Engineering Univ. Grenoble Alpes \\ \{ahmad.hably\}@gipsa-lab.fr
}

\begin{abstract}
This paper presents a comparison between Mixed strategist dynamics (MSD) and a generalized evolutionary game dynamics known as Intersection Escort Evolutionary Dynamics (IEED). Through illustrative examples, it will be shown that IEED exhibits a computational advantage over MSD especially for high order and non-homogeneous constraints for the variables of the problem under study.
\end{abstract}

\section{INTRODUCTION}

Evolutionary game theory (EGT) models interactions between portions of a population which try to maximize their wellness. Wellness is considered as the payoff obtained for using a certain combination of strategies. Portions of the population with strategies giving higher than average payoff will expand quicker and the proportion of these portions will tend to grow. [1]. In Reference [2], the advantages of using EGT in engineering applications are presented. Its application in several engineering examples such as smart lighting and optimal economic dispatch in micro-grids has been presented. Another approach has been used for distributed optimization in [3], for wireless networks in [4], and for load management of electric vehicle fleets in [5], [6]. EGT is used in [7] for automating node clustering and nominations of cluster heads, in order to achieve cluster stability in Vehicular Ad hoc Networks, and to analyze advanced persistent threats against cloud storage in [8]. In [9], EGT has been employed to analyze strategic choice between two competing alternatives having their own business case in employing upward consumption flexibility to alleviate problems caused by wind generation and renewable energy support schemes.

Replicator dynamics (RD) is a simple model of a biological evolutionary process. This model assumes the increase/evolution of a proportion of the population, hosting a replicator, at a certain rate related to this fraction of the hosting population and to the difference between of the hosts' fitness and the mean fitness [10], [1]. These dynamics is modelled as a system ordinary differential equations. As mentioned by [11], in RD a mixed strategy is a population state and each component of this mixed strategy represents a population share of individuals using a corresponding pure strategy. RD has been successfully applied as a tool to solve NP-hard combinatorial optimization problems [10], [12]. By replacing some terms in RD system with incentive functions, as proposed in [13], several models of evolutionary dynamics can be generated. These results are used in [14] to get a natural generalization of evolutionary stable states and a suitable version of relative entropy that can be used as a Lyapunov function to prove stability. In [6], generalized evolutionary dynamics for limited hosting capacities have been employed. It is called Intersection Escort Evolutionary Dynamics (IEED) and it is an extension to Escort Evolutionary Dynamics of [15]. These dynamics describes the evolution of portions of a population following $K$ different pure strategies according to the benefit they provide. It was originally employed for a distributed optimization application for electric vehicle fleet charging management. In order to apply the desirable features of RD for a general resource allocation problem, a different extension of RD has to be introduced. Here, populations will follow mixed strategies instead of pure strategies and this are known as Mixed strategist dynamics (MSD) [1].

In this present paper, a comparison between MSD and IEED will be given. After an introduction of MSD in section II, with an illustrative example using Rock-PaperScissors game, IEED will be presented in section III. A detailed example is given in section IV in order to compare these two dynamics. The paper ends with some conclusions in section V.

\section{Mixed Strategist DynAmics}

In this section, Mixed Strategist Dynamics (MSD) as described in [1], [16] will be introduced. The MSD represents the evolution of portions of a population follow- 
ing $M$ different Mixed Strategies (MSs). These MSs are defined from $K$ original pure strategies, according to the benefit they provide. The continuous time MSD can then be described by,

$$
\dot{y}_{m}=y_{m}\left(g_{m}(\mathbf{y})-\bar{g}(\mathbf{y})\right)
$$

where $\mathbf{g}(\cdot)=\left[g_{1}(\cdot), \cdots, g_{m}(\cdot), \cdots, g_{M}(\cdot)\right]^{\mathrm{T}}$ defines the payoff landscape for pure strategies, and $\bar{g}(\mathbf{y})$ represents the mean payoff for the population. If a matrix $\mathbf{C}$ gathering predefined mixed strategies as column vectors $\mathbf{c}_{m}$ is defined, then the elements $c_{k, m}$ of the column vector $\mathbf{c}_{m}$ are the coefficients in a convex linear combination of pure strategies, i.e., $c_{k, m} \geq 0, \sum_{k} c_{k, m}=1$. If $\mathbf{y}$ represents the population distribution per mixed strategies, the corresponding distribution per pure strategies $\mathbf{x}$ can then be written by,

$$
\mathbf{x}=\mathbf{C y}
$$

With this notation, let us focus on the original payoff landscape $\mathbf{f}(\cdot)=\left[f_{1}(\cdot), \cdots, f_{k}(\cdot), \cdots, f_{K}(\cdot)\right]^{\mathrm{T}}$ defined for pure strategies. The argument of this payoff landscape is the distribution per pure strategies $\mathbf{x}$. The payoff obtained for a portion of a population using a mixed strategy $\mathbf{c}_{m}$ is determined by the expected value associated to the mixed strategy and the payoff associated to the population distribution, i.e., $g_{m}=\mathbf{c}_{m}^{\mathrm{T}} \mathbf{f}(\mathbf{x})$. In other words, the relation between payoff landscapes $\mathbf{f}(\cdot)$ and $\mathbf{g}(\cdot)$ is given by,

$$
\mathbf{g}(\mathbf{y})=\mathbf{C}^{\mathrm{T}} \mathbf{f}(\mathbf{x})=\mathbf{C}^{\mathrm{T}} \mathbf{f}(\mathbf{C y}) .
$$

$\mathbf{f}(\cdot)$ defines the payoff landscape for pure strategies, and $\bar{g}(\mathbf{y})$ represents de mean payoff for the population. This leads to an equivalence in the definitions of mean payoffs using both landscapes $\mathbf{f}(\cdot)$ and $\mathbf{g}(\cdot)$, i.e.,

$$
\bar{f}(\mathbf{x})=\mathbf{x}^{\mathrm{T}} \mathbf{f}(\mathbf{x})=\mathbf{y}^{\mathrm{T}} \mathbf{C}^{\mathrm{T}} \mathbf{f}(\mathbf{C y})=\mathbf{y}^{\mathrm{T}} \mathbf{g}(\mathbf{y})=\bar{g}(\mathbf{y})
$$

Moreover, the MSD can be defined in terms of the original payoff landscape $\mathbf{f}(\cdot)$ as,

$$
\dot{y}_{m}=y_{m}\left(\left(\mathbf{C}^{\mathrm{T}} \mathbf{f}(\mathbf{C y})\right)_{m}-\bar{g}(\mathbf{y})\right) .
$$

Given that eq.(1) sets a lower barrier on zero to $y_{m}, \mathbf{y}$ will always lie within the simplex $\Delta^{M}=\left\{\mathbf{y} \in \mathbb{R}^{M}\right.$ : $\left.y_{m} \geq 0, \sum_{m} y_{m}=1\right\}$. Consequently, $\mathbf{x}$ will also always lie within the simplex $\Delta^{K}=\left\{\mathbf{x} \in \mathbb{R}^{K}: x_{k} \geq\right.$ $\left.0, \sum_{k} x_{k}=1\right\}$. Furthermore, considering the definition of MSs in $\mathbf{C}$, the population distribution $\mathbf{x}$ will always lie in a subset $\Theta^{M} \in \Delta^{K}$ defined by the following convex hull,

$$
\Theta^{M}=\left\{\mathbf{x}=\mathbf{C y} \in \mathbb{R}^{K}: y_{m} \geq 0, \sum_{m} y_{m}=1\right\} .
$$

Taking advantage of this, it is possible to confine the trajectories of the population state vector $\mathbf{x}$ to a reduced subset of $\Delta^{K}$ and introduce hosting capacity limitations for pure strategies.

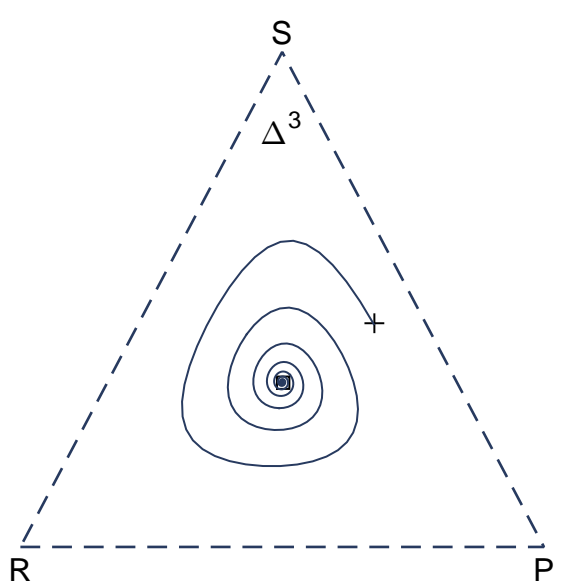

Fig. 1. Simplex $\Delta^{3}$ in $\mathbb{R}^{3}$. Vertices of convex sets $\Theta^{6}$ and $\Theta^{3}$ represent mixed strategies $\mathbf{c}_{m}$. In blue, trajectory when the set of MSs is exactly the set of pure strategies.

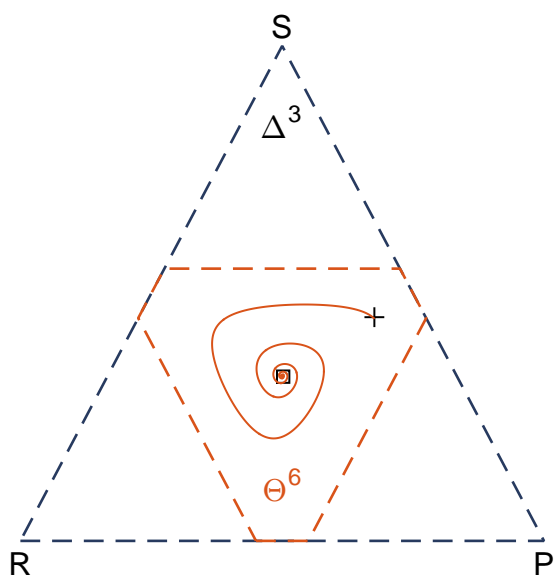

Fig. 2. Simplex $\Delta^{3}$ in $\mathbb{R}^{3}$. Vertices of convex sets $\Theta^{6}$ and $\Theta^{3}$ represent mixed strategies $\mathbf{c}_{m}$. In orange, trajectories for the RPS game with different sets of MSs.

\section{A. Example with the Rock-Paper-Scissors game}

Let us take a look on the following classic RockPaper-Scissors example [1]. The payoff landscape for pure strategies is described by,

$$
\mathbf{f}(\mathbf{x})=\left[\begin{array}{ccc}
0 & -a & b \\
b & 0 & -a \\
-a & b & 0
\end{array}\right] \mathbf{x}
$$

where $a, b>0$. This game has a global attractor on $\hat{\mathbf{x}}=$ $[1 / 3,1 / 3,1 / 3]^{\mathrm{T}}$ for $a<b$.

Let us define three subsets $\Theta^{M}$ represented by three different sets of MSs, containing the same global attractor. Trajectories followed by the MSD for each set of MSs, are presented on Figs. 1-3. The dark blue trajectory results when the set of MSs is identical to the set of pure strategies, i.e., $\mathbf{C}=\mathbf{I}$ the identity. The orange portrait shows the resulting trajectory for a set of 6 MSs located in pairs at 


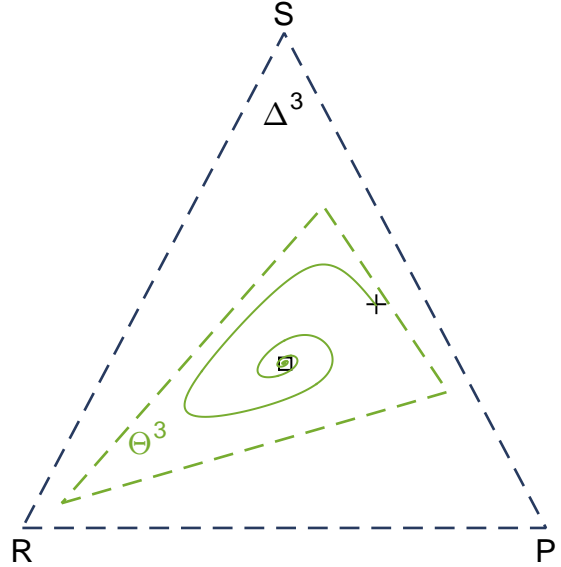

Fig. 3. Simplex $\Delta^{3}$ in $\mathbb{R}^{3}$. Vertices of convex sets $\Theta^{6}$ and $\Theta^{3}$ represent mixed strategies $\mathbf{c}_{m}$. In green, trajectories for the RPS game with different sets of MSs.

each of the three boundaries of the simplex, as it is shown. The third trajectory, in green, results when $\mathbf{C}$ is defined by $3 \mathrm{MSs}$ placed inside the simplex. Both sets of MSs are given in,

$$
\begin{gathered}
\mathbf{C}_{\Theta^{6}}=\left[\begin{array}{cccccc}
0.55 & 0.45 & 0 & 0 & 0.45 & 0.55 \\
0.45 & 0.55 & 0.55 & 0.45 & 0 & 0 \\
0 & 0 & 0.45 & 0.55 & 0.55 & 0.45
\end{array}\right] \\
\mathbf{C}_{\Theta^{3}}=\left[\begin{array}{cccc}
0.9 & 0.05 & 0.1 \\
0.05 & 0.675 & 0.25 \\
0.05 & 0.275 & 0.65
\end{array}\right] .
\end{gathered}
$$

The initial distribution in the three cases is $\mathbf{x}=$ $[0.1,0.45,0.45]^{\mathrm{T}}$. For the subset $\Theta^{3}$, this initial distribution corresponds to a distribution per mixed strategies given by $\mathbf{y}(0)=[0.0304,0.4848,0.4848]$. For the subset $\Theta^{6}$, it corresponds to an initial distribution per mixed strategies $\mathbf{y}(0)=[0.05,0.05,0.4,0.4,0.05,0.05]$.

In this present paper, the most interesting case is represented by the subset $\Theta^{6}$. In this case, a limited hosting capacity of $55 \%$ of the population per pure strategy is represented. In other words, the population is still playing a Rock-Paper-Scissors game but the MSs definition has introduced a constraint in the number of individuals that can use each of the original pure strategies.

Keeping these definitions in mind, the following section describes the Intersection Escort Evolutionary Dynamics (IEED) previously introduced in [6] and its role in the representation of limited hosting capacities.

\section{INTERSECTION ESCORT EVOLUTIONARY DYNAMICS}

Intersection escort evolutionary dynamics (IEED) has the particular property that it can be employed to describe the intersection of two simplices representing lower and upper boundaries as it will be demonstrated in this section. The continuous time IEED can be described by,

$$
\dot{x}_{k}=\alpha_{k} \beta_{k}\left(f_{k}(\mathbf{x})-\bar{f}_{\alpha \beta}(\mathbf{x})\right),
$$

where $\mathbf{f}(\cdot)=\left[f_{1}(\cdot), \cdots, f_{k}(\cdot), \cdots, f_{K}(\cdot)\right]^{\mathrm{T}}$ defines the payoff landscape for pure strategies, and $\bar{f}_{\alpha \beta}(\mathbf{x})$ represents the weighted average payoff for the population depending on values of functions $\alpha_{k}$ and $\beta_{k}$. These functions depend on the population distribution $\mathbf{x}$, and the boundaries or, in this case, the hosting capacities for each pure strategy.

For reference purposes let us call the product $\phi_{k}=$ $\alpha_{k} \beta_{k}$ the Escort function to match with the original definition of [15]. Using the normalized vector of escort functions,

$$
\hat{\phi}=\frac{1}{\sum_{k} \alpha_{k} \beta_{k}}\left[\alpha_{1} \beta_{1}, \cdots, \alpha_{k} \beta_{k}, \cdots, \alpha_{K} \beta_{K}\right]^{\mathrm{T}},
$$

the weighted average payoff for the population $\bar{f}_{\alpha \beta}(\mathbf{x})$ is computed as the expected value,

$$
\bar{f}_{\alpha \beta}(\mathbf{x})=\hat{\phi}^{\mathrm{T}} \mathbf{f}(\mathbf{x}) .
$$

\section{A. Definition of functions $\alpha_{k}$ and $\beta_{k}$}

Let us consider the scenario where the hosting capacities for each pure strategy are defined by a subset $\Psi^{K}$ of the simplex $\Delta^{K}$ defined by,

$$
\Psi^{K}=\left\{\mathbf{x} \in \mathbb{R}^{K}: x_{k}^{l o} \leq x_{k} \leq x_{k}^{u p}, \quad \sum_{k} x_{k}=1\right\},
$$

where $x_{k}^{l o}$ and $x_{k}^{u p}$ are the corresponding upper and lower boundaries of the hosting capacity for each portion $x_{k}$ of the normalized population. This definition can be rewritten as the intersection $\Psi^{K}=\Delta_{l o}^{K} \cap \Delta_{u p}^{K}$ of two simplices defined by,

$$
\begin{aligned}
\Delta_{l o}^{K} & =\left\{\mathbf{x} \in \mathbb{R}^{K}: x_{k} \geq x_{k}^{l o}, \quad \sum_{k} x_{k}=1\right\}, \\
\Delta_{u p}^{K} & =\left\{\mathbf{x} \in \mathbb{R}^{K}: x_{k} \leq x_{k}^{u p}, \quad \sum_{k} x_{k}=1\right\},
\end{aligned}
$$

Following a similar principle as in Sect. II, these simplices can also be expressed as the following convex hulls,

$$
\begin{gathered}
\Delta_{l o}^{K}=\left\{\mathbf{x}=\mathbf{C}_{l o} \beta: \beta \in \mathbb{R}^{K}, \beta_{k} \geq 0, \quad \sum_{k} \beta_{k}=1\right\}, \\
\Delta_{u p}^{K}=\left\{\mathbf{x}=\mathbf{C}_{u p} \alpha: \alpha \in \mathbb{R}^{K}, \alpha_{k} \geq 0, \quad \sum_{k} \alpha_{k}=1\right\},
\end{gathered}
$$

where the column vectors of matrices $\mathbf{C}_{l o}$ and $\mathbf{C}_{u p}$ are the vertices of the simplices $\Delta_{l o}^{K}$ and $\Delta_{u p}^{K}$ respectively. Column vectors of these matrices are two separate sets of vectors that form two bases for $\mathbb{R}^{K}$. In fact, if the lower boundaries are all zeros, $\mathbf{C}_{l o}$ becomes the identity, i.e., the standard basis for $\mathbb{R}^{K}$, and $\Delta_{l o}^{K}$ becomes the standard simplex.

As it can be inferred, matrices $\mathbf{C}_{l o}$ and $\mathbf{C}_{u p}$ must be somehow related to upper and lower boundaries. In fact, 
finding these matrices is a simple procedure, and even if it is not necessary to find them explicitly for the IEED. They are illustrated for the definition of functions $\alpha_{k}$ and $\beta_{k}$. Let us consider square matrices $\mathbf{X}_{l o}$ and $\mathbf{X}_{u p}$ defined with the boundaries as,

$$
\begin{aligned}
\mathbf{X}_{l o} & =\left[\begin{array}{cccc}
x_{1}^{l o} & x_{1}^{l o} & \cdots & x_{1}^{l o} \\
\vdots & \vdots & & \vdots \\
x_{k}^{l o} & x_{k}^{l o} & \cdots & x_{k}^{l o} \\
\vdots & \vdots & & \vdots \\
x_{K}^{l o} & x_{K}^{l o} & \cdots & x_{K}^{l o}
\end{array}\right] \\
\mathbf{X}_{u p}= & {\left[\begin{array}{cccc}
x_{1}^{u p} & x_{1}^{u p} & \cdots & x_{1}^{u p} \\
\vdots & \vdots & & \vdots \\
x_{k}^{u p} & x_{k}^{u p} & \cdots & x_{k}^{u p} \\
\vdots & \vdots & & \vdots \\
x_{K}^{u p} & x_{K}^{u p} & \cdots & x_{K}^{u p}
\end{array}\right], }
\end{aligned}
$$

and scalars $\sigma_{l o}$ and $\sigma_{u p}$ also defined with the boundaries as,

$$
\sigma_{l o}=1-\sum_{k} x_{k}^{l o}, \quad \sigma_{u p}=1-\sum_{k} x_{k}^{l o} .
$$

With these matrices, vertices of the upper and lower simplices can be found with the following expressions,

$$
\mathbf{C}_{l o}=\mathbf{X}_{l o}-\sigma_{l o} \mathbf{I}, \quad \mathbf{C}_{u p}=\mathbf{X}_{u p}-\sigma_{u p} \mathbf{I},
$$

where $\mathbf{I}$ is the identity matrix. From the convex hull definitions of the upper and lower simplices, let us recall that $\mathbf{x}=\mathbf{C}_{l o} \beta$, and $\mathbf{x}=\mathbf{C}_{u p} \alpha$. Hence,

$$
\begin{aligned}
& \beta=\mathbf{C}_{l o}^{-1} \mathbf{x}=\frac{1}{\sigma_{l o}}\left(\mathbf{I}-\mathbf{X}_{l o}\right) \mathbf{x}, \\
& \alpha=\mathbf{C}_{u p}^{-1} \mathbf{x}=\frac{1}{\sigma_{u p}}\left(\mathbf{I}-\mathbf{X}_{u p}\right) \mathbf{x} .
\end{aligned}
$$

Having in mind that the population distribution $\mathbf{x}$ lies in the hyper-plane $\sum_{k} x_{k}=1$, these expressions can be further simplified to the following functions of $x_{k}$,

$$
\begin{aligned}
\beta_{k} & =\frac{1}{\sigma_{l o}}\left(x_{k}-x_{k}^{l o}\right), \\
\alpha_{k} & =\frac{1}{\sigma_{u p}}\left(x_{k}-x_{k}^{u p}\right),
\end{aligned}
$$

which are the expressions that define the escort functions in the IEED. Functions $\beta_{k}$ and $\alpha_{k}$ provide direct information of $x_{k}$ approaching $x_{k}^{l o}$ or $x_{k}^{u p}$ respectively. Multiple elements should be highlighted from these definitions. First, It can be noticed that scalar $\sigma_{l o}$ is always positive, while scalar $\sigma_{u p}$ is always negative. If these conditions are not fulfilled, this means that boundaries are too restrictive and constraints are not feasible. To illustrate this, let us take a look on eqs.(16) and assume a positive $\sigma_{u p}$. Consequently $\sum_{k} x_{k}^{u p}<1$ which means that upper constraints are too low to allow $\sum_{k} x_{k}$ to be the unity. A similar reasoning applies for a negative $\sigma_{l o}$ and $\sum_{k} x_{k}^{l o}>1$.

On the other hand, a consequence of $\sigma_{l o}>0$, and $\sigma_{u p}<0$, is that $\beta_{k}$ is monotonically increasing while $\alpha_{k}$ is monotonically decreasing. Moreover, $\beta_{k}=0$ when $x_{k}=x_{k}^{l o}$, and $\alpha_{k}=0$ when $x_{k}=x_{k}^{u p}$. This imposes two barriers on the dynamics as described by eq. (6). It is also useful to notice that $\beta_{k}=1$ when $x_{k}=\sigma_{l o}+x_{k}^{l o}$, and $\alpha_{k}=1$ when $x_{k}=\sigma_{u p}+x_{k}^{u p}$. These last cases represent the vertices $k$ of the lower and upper simplices respectively.

A final important fact to notice is that, if boundaries are feasible, the escort function represented by the product of functions $\alpha_{k}$ and $\beta_{k}$ is always positive for all $k$.

\section{B. Stability}

In the definitions of [15], escort functions were imposed to be positive and monotonically increasing. However, for the IEED functions $\alpha_{k}$ and $\beta_{k}$ were demonstrated to be positive, but one of them decreases as the other increases. As a result, the product is not monotonically increasing but is still positive. Authors of [17] proved the stability of the escort evolutionary dynamics with positive monotonically increasing escort functions. Even if it is out of the scope of this paper, it is possible to prove that the following divergence-like function,

$$
\begin{aligned}
L(\mathbf{x})=\sum_{k=1}^{K} \frac{\sigma_{u p} \sigma_{l o}}{x_{k}^{u p}-x_{k}^{l o}} & \left\{\left(\hat{x}_{k}-x_{k}^{u p}\right) \log \left(\frac{\hat{x}_{k}-x_{k}^{u p}}{x_{k}-x_{k}^{u p}}\right)\right. \\
& \left.-\left(\hat{x}_{k}-x_{k}^{l o}\right) \log \left(\frac{\hat{x}_{k}-x_{k}^{l o}}{x_{k}-x_{k}^{l o}}\right)\right\},
\end{aligned}
$$

is a Lyapunov function for the IEED, with equilibrium state $\hat{\mathbf{x}}$.

\section{Example with the Rock-Paper-Scissors game}

Let us take a look again on the Rock-Paper-Scissors example from Sect. III. This time the hosting capacities will be assumed to be bounded up and down for each of the pure strategies. Let us consider $\mathbf{x} \in \Delta_{l o}^{3}=\left\{x_{1} \geq\right.$ $\left.0.05, x_{2} \geq 0.1, x_{3} \geq 0.075, x_{1}+x_{2}+x_{3}=1\right\}$. Given these lower constraints, the resulting matrix $\mathbf{C}_{l o}$ computed with (17) is,

$$
\begin{aligned}
\mathbf{C}_{l o}=\mathbf{X}_{l o}+\sigma_{l o} \mathbf{I} & =\left[\begin{array}{ccc}
0.05 & 0.05 & 0.05 \\
0.1 & 0.1 & 0.1 \\
0.075 & 0.075 & 0.075
\end{array}\right] \\
+0.775\left[\begin{array}{lll}
1 & 0 & 0 \\
0 & 1 & 0 \\
0 & 0 & 1
\end{array}\right] & \\
& =\left[\begin{array}{ccc}
0.825 & 0.05 & 0.05 \\
0.1 & 0.875 & 0.1 \\
0.075 & 0.075 & 0.85
\end{array}\right],
\end{aligned}
$$


while $\beta_{k}$ functions are,

$\beta_{1}=\frac{x_{1}-0.05}{0.775}, \quad \beta_{2}=\frac{x_{2}-0.1}{0.775}, \quad \beta_{3}=\frac{x_{3}-0.075}{0.775}$.

As it was explained, columns of $\mathbf{C}_{l o}$ are the vertices of the simplex $\Delta_{l o}^{K}$. Let us take a look now on upper boundaries on each dimension defined by $\mathbf{x} \in \Delta_{\text {up }}^{3}=$ $\left\{x_{1} \leq 0.4, x_{2} \leq 0.5, x_{3} \leq 0.75, x_{1}+x_{2}+x_{3}=1\right\}$.

Given these constraints, the resulting $\mathbf{C}_{u p}$ matrix is,

$$
\begin{aligned}
\mathbf{C}_{u p}=\mathbf{X}_{u p}+\sigma_{u p} \mathbf{I} & =\left[\begin{array}{ccc}
0.4 & 0.4 & 0.4 \\
0.5 & 0.5 & 0.5 \\
0.75 & 0.75 & 0.75
\end{array}\right] \\
-0.65\left[\begin{array}{lll}
1 & 0 & 0 \\
0 & 1 & 0 \\
0 & 0 & 1
\end{array}\right] & \\
& =\left[\begin{array}{ccc}
-0.25 & 0.4 & 0.4 \\
0.5 & -0.15 & 0.5 \\
0.75 & 0.75 & 0.1
\end{array}\right],
\end{aligned}
$$

while $\alpha_{k}$ functions are,

$\alpha_{1}=\frac{-x_{1}+0.4}{0.65}, \quad \alpha_{2}=\frac{-x_{2}+0.5}{0.65}, \quad \alpha_{3}=\frac{-x_{3}+0.7 \text { ? }}{0.65}$

Again, the columns of $\mathbf{C}_{u p}$ are the vertices of the simplex $\Delta_{u p}^{3}$ as it was explained before. With these expressions in mind, the resulting escort functions are,

$$
\begin{gathered}
\phi_{1}=\frac{\left(x_{1}-0.05\right)\left(0.4-x_{1}\right)}{0.50375}, \phi_{2}=\frac{\left(x_{2}-0.1\right)\left(0.5-x_{2}\right)}{0.50375}, \\
\phi_{3}=\frac{\left(x_{3}-0.075\right)\left(0.75-x_{3}\right)}{0.50375}
\end{gathered}
$$

which are positive valued for points in the intersection $\mathbf{x} \in$ $\Delta_{l o}^{3} \cap \Delta_{u p}^{3}$. If $\left\{\phi_{1}, \phi_{2}, \phi_{3}\right\}$ are positive, then it is true that $\left\{\alpha_{1}, \alpha_{2}, \alpha_{3}, \beta_{1}, \beta_{2}, \beta_{3}\right\}$ are positive as well. Given that the ED guarantees $\left\{x_{1}+x_{2}+x_{3}=1\right\}$, then,

$\alpha_{1}+\alpha_{2}+\alpha_{3}=\frac{-x_{1}+0.75-x_{2}+0.4-x_{3}+0.5}{0.65}=1$,

$\beta_{1}+\beta_{2}+\beta_{3}=\frac{x_{1}-0.075+x_{2}+0.05+x_{3}-0.1}{0.875}=1$,

which are consequently always satisfied as well. Then, any trajectory followed by the IEED with escort functions $\left\{\phi_{1}, \phi_{2}, \phi_{3}\right\}$, satisfies both sets $\left\{\alpha_{1}, \alpha_{2}, \alpha_{3} \geq 0, \alpha_{1}+\right.$ $\left.\alpha_{2}+\alpha_{3}=1\right\}$ and $\left\{\beta_{1}, \beta_{2}, \beta_{3} \geq 0, \beta_{1}+\beta_{2}+\beta_{3}=\right.$ $1\}$. Consequently, all the escort functions also have upper limits at $\left\{\alpha_{1}, \alpha_{2}, \alpha_{3}, \beta_{1}, \beta_{2}, \beta_{3} \leq 1\right\}$ and the intersections among the following intervals are fulfilled,

$$
\begin{gathered}
0 \leq \alpha_{1} \leq 1, \quad 0 \leq \alpha_{2} \leq 1, \quad 0 \leq \alpha_{3} \leq 1 \\
0 \leq \frac{-x_{1}+0.4}{0.65} \leq 1 \\
0 \leq \frac{-x_{2}+0.5}{0.65} \leq 1, \quad 0 \leq \frac{-x_{3}+0.75}{0.65} \leq 1, \\
-0.25 \leq x_{2} \leq 0.4, \quad-0.15 \leq x_{3} \leq 0.5, \\
0.1 \leq x_{1} \leq 0.75
\end{gathered}
$$

$$
\begin{gathered}
0 \leq \beta_{1} \leq 1, \quad 0 \leq \beta_{2} \leq 1, \quad 0 \leq \beta_{3} \leq 1 \\
0 \leq \frac{x_{1}-0.05}{0.775} \leq 1, \quad 0 \leq \frac{x_{2}-0.1}{0.775} \leq 1 \\
0 \leq \frac{x_{3}-0.075}{0.775} \leq 1 \\
0.05 \leq x_{1} \leq 0.825
\end{gathered}
$$$$
0.1 \leq x_{2} \leq 0.875, \quad 0.075 \leq x_{3} \leq 0.85
$$

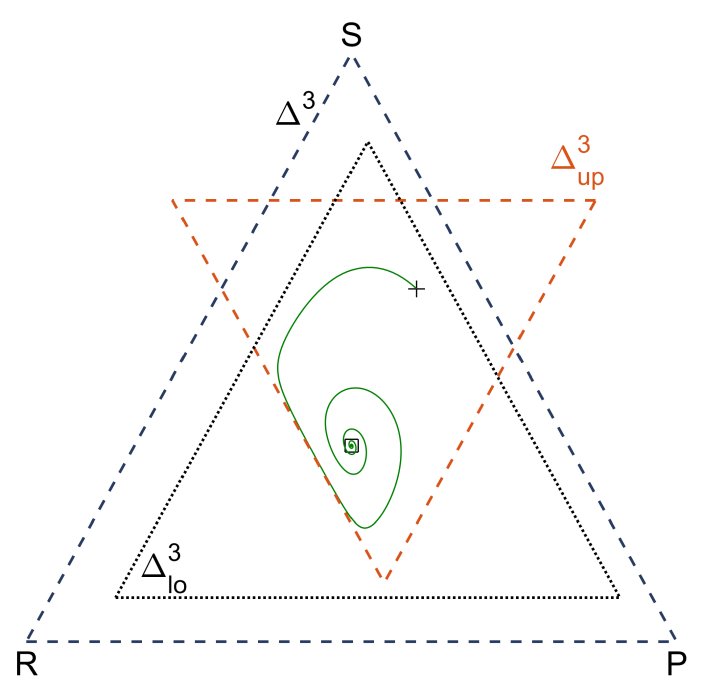

Fig. 4. Simplices $\Delta^{3}, \Delta_{l o}^{3}$, and $\Delta_{u p}^{3}$ in $\mathbb{R}^{3}$. In green, trajectory in the intersection of the upper and lower simplices. In this example $a=1$ and $b=2$.

With the standard simplex as reference, the upper and lower simplices are plotted on Fig. 4 for this example. It is possible to see the portrait of a trajectory arriving to the global attractor, while boundaries are being respected during the whole evolution.

\section{AN EXAMPLE OF COMPARISON BETWEEN MSD AND IEED}

In order to compare the MSD and the IEED, let us consider the example of Figs. 5-6. In this case, the payoff landscape is defined as the euclidean gradient of the potential function $F(\mathbf{x})=-\left(x_{1}-13 / 20\right)^{2}-\left(x_{2}-\right.$ 
a)

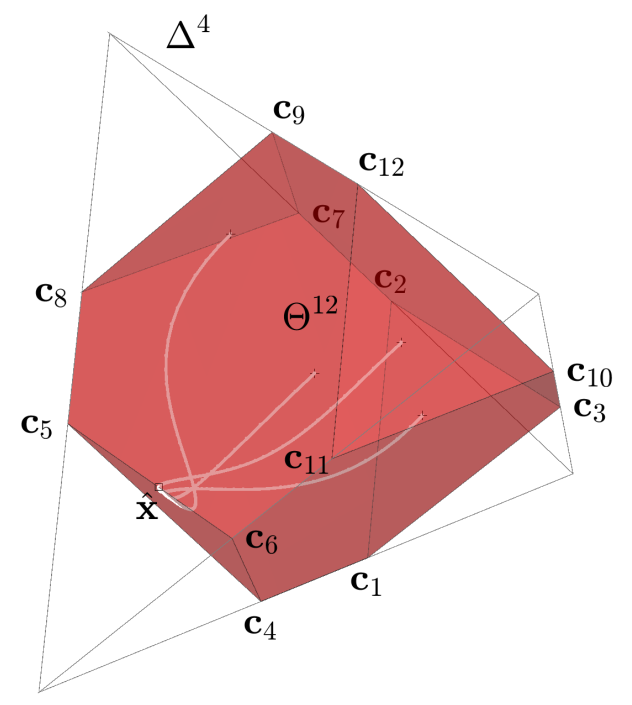

Fig. 5. Example of comparison between MSD and IEED. The intersection between the standard simplex $\Delta^{4}$ and a simplex of upper constraints $\Delta_{u p}^{4}$, both in $\mathbb{R}^{4}$, is shown. Representation for the MSD approach: 12 vertices (12 mixed strategies) define the convex hull of the intersection.

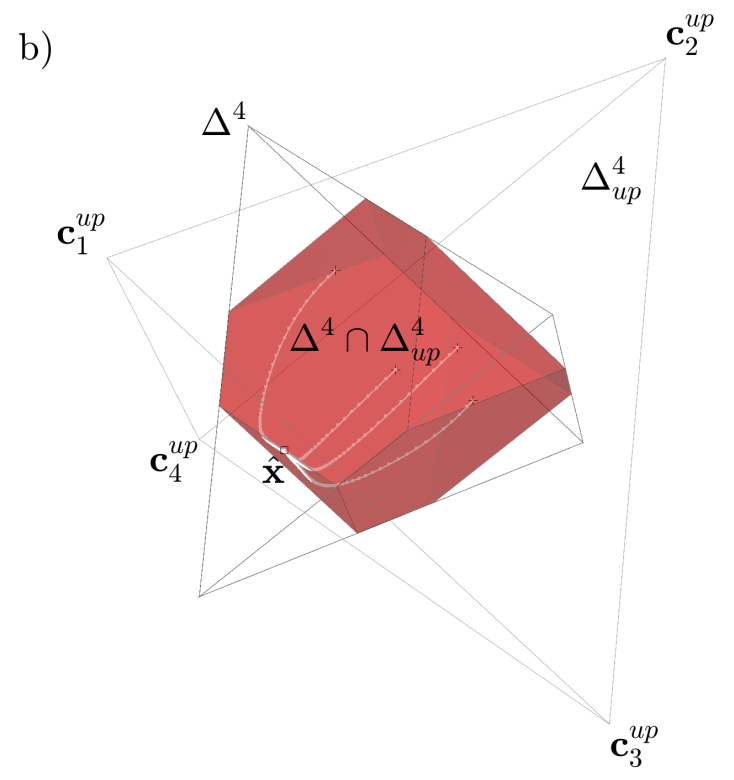

Fig. 6. Example of comparison between MSD and IEED. The intersection between the standard simplex $\Delta^{4}$ and a simplex of upper constraints $\Delta_{u p}^{4}$, both in $\mathbb{R}^{4}$, is shown. For the IEED approach: the intersection is represented without knowledge of its vertices.

$40 / 20)^{2}-\left(x_{3}-17 / 20\right)^{2}-\left(x_{4}-18 / 20\right)^{2}$, for $\mathbf{x} \in \mathbb{R}^{4}$. This potential function has an unconstrained maximum at $\hat{\mathbf{x}}^{+}=[13 / 20,40 / 20,17 / 20,18 / 20]^{\mathrm{T}}$, and if it is constrained to points $\mathbf{x} \in \Theta^{12}=\left\{0 \leq x_{1}, x_{2}, x_{3}, x_{4} \leq\right.$ $\left.0.6, x_{1}+x_{2}+x_{3}+x_{4}=1\right\}$, then the feasible maximum is $\hat{\mathbf{x}}=[0,0.6,0.175,0.225]^{\mathrm{T}}$.

The feasible region in this case is defined by upper and lower constraints on each of the four variables, and it can represented by the vertices of the intersection set between the standard simplex (defined by lower constraints) $\Delta^{4}=$ $\left\{x_{1}, x_{2}, x_{3}, x_{4} \geq 0, x_{1}+x_{2}+x_{3}+x_{4}=1\right\}$, and the simplex of upper constraints $\Delta_{u p}^{4}=\left\{x_{1}, x_{2}, x_{3}, x_{4} \leq\right.$ $\left.0.6, x_{1}+x_{2}+x_{3}+x_{4}=1\right\}$. Given that these constraints are homogeneous (i.e., they are shared by all the variables), the vertices of the intersection can be easily found as all the possible permutations of the vector $\mathbf{c}_{1}=[0.6,0.4,0,0]^{\mathrm{T}}$, which are listed as the columns of the following matrix,

$$
\mathbf{C}_{\Theta^{12}}=\left[\begin{array}{rrrr}
0.6 & 0.4 & 0 & 0 \\
0.6 & 0 & 0.4 & 0 \\
0.6 & 0 & 0 & 0.4 \\
0.4 & 0.6 & 0 & 0 \\
0 & 0.6 & 0.4 & 0 \\
0 & 0.6 & 0 & 0.4 \\
0.4 & 0 & 0.6 & 0 \\
0 & 0.4 & 0.6 & 0 \\
0 & 0 & 0.6 & 0.4 \\
0.4 & 0 & 0 & 0.6 \\
0 & 0.4 & 0 & 0.6 \\
0 & 0 & 0.4 & 0.6
\end{array}\right]^{T}
$$

These column vectors are the mixed strategies employed by the MSD. Fig. 5 shows a representation of the simplex $\Delta^{4}$, and the intersection $\Theta^{12}$ along with the corresponding defining vertices. $\Delta^{4}$ can be represented in $\mathbb{R}^{3}$ since the hyper-plane $\left\{x_{1}+x_{2}+x_{3}+x_{4}=1\right\}$ is a 3 -dimensional subspace of $\mathbb{R}^{4}$ and the simplex is a portion of this subspace. Furthermore, four different trajectories followed by the MSD, arriving to the equilibrium state, are presented. The resulting final distributions assign $43.76 \%$ of the population to mixed strategy $\mathbf{c}_{5}$, and the $56.24 \%$ left, to mixed strategy $\mathbf{c}_{6}$, which results in the previously mentioned local feasible equilibrium state $\hat{\mathbf{x}}=[0,0.6,0.175,0.225]^{\mathrm{T}}$.

On the other hand, Fig. 6 shows the same example, but applying the proposed IEED instead. In this case, the escort functions assigned to each of the four variables are equal to,

$$
\phi_{k}=\alpha_{k} \beta_{k}=\frac{\left(x_{k}-0.6\right)}{-1.4} \frac{\left(x_{k}-0\right)}{1}=\frac{x_{k}\left(0.6-x_{k}\right)}{1.4},
$$


a)

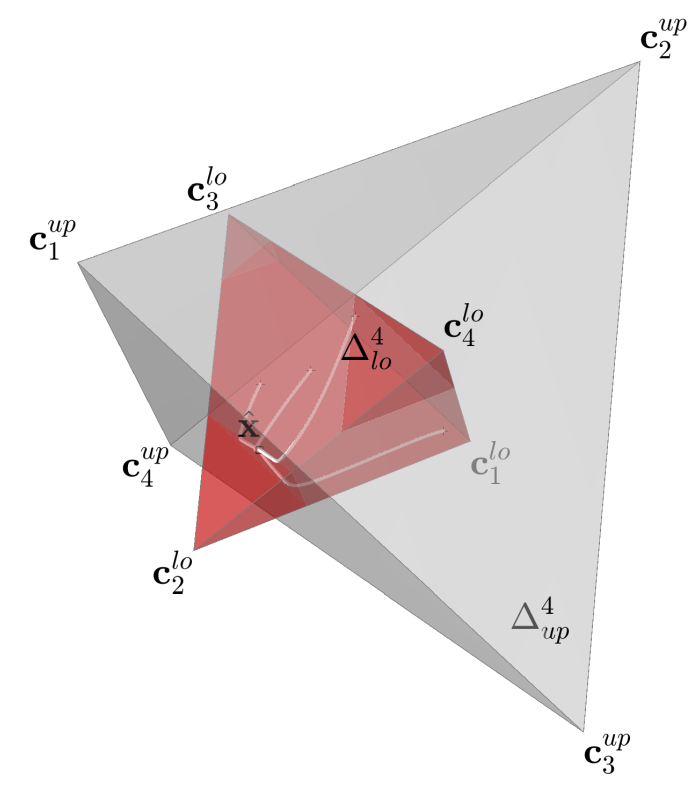

Fig. 7. An example of simplices $\Delta_{u p}^{4}$, and $\Delta_{l o}^{4}$, both in $\mathbb{R}^{4}$. Example of intersection subset defined by 10 vertices.

and the resulting matrix $\mathbf{C}_{u p}$ computed with (17) is,

$$
\begin{aligned}
\mathbf{C}_{u p}=\mathbf{X}_{u p}+\sigma_{u p} \mathbf{I} & =\left[\begin{array}{cccc}
0.6 & 0.6 & 0.6 & 0.6 \\
0.6 & 0.6 & 0.6 & 0.6 \\
0.6 & 0.6 & 0.6 & 0.6 \\
0.6 & 0.6 & 0.6 & 0.6
\end{array}\right] \\
& -1.4\left[\begin{array}{cccc}
1 & 0 & 0 & 0 \\
0 & 1 & 0 & 0 \\
0 & 0 & 1 & 0 \\
0 & 0 & 0 & 1
\end{array}\right] \\
& =\left[\begin{array}{cccc}
-0.8 & 0.6 & 0.6 & 0.6 \\
0.6 & -0.8 & 0.6 & 0.6 \\
0.6 & 0.6 & -0.8 & 0.6 \\
0.6 & 0.6 & 0.6 & -0.8
\end{array}\right] .
\end{aligned}
$$

Matrix $\mathbf{C}_{l o}=\mathbf{I}$ since $\Delta_{l o}^{4}$ is the standard simplex $\Delta^{4}$ (it can be verified applying eq. (17) as well). Column vectors of $\mathbf{C}_{u p}$ are the vertices of the simplex $\Delta_{u p}^{4}$ represented in Fig. 6. Finding these vertices is a much simpler procedure than listing the 12 permutations that define the vertices of the intersection for the MSD approach. Moreover, in the IEED approach, finding $\mathbf{C}_{u p}$ is not needed (it is only needed for purposes of graphical representation), because the IEED only employs the parameters of the escort functions (i.e. the constraining limits, $x_{k}^{l o}, x_{k}^{u p}$, $\sigma_{u p}$, and $\sigma_{l o}$ ). Fig. 6 also shows four different trajectories starting from the same initial distributions than the case with MSD, and arriving to the same equilibrium state $\hat{\mathbf{x}}=$ $[0,0.6,0.175,0.225]^{\mathrm{T}}$. Both approaches produce different trajectories inside the feasible region, but the equilibrium state is asymptotically reached by both of them. b)

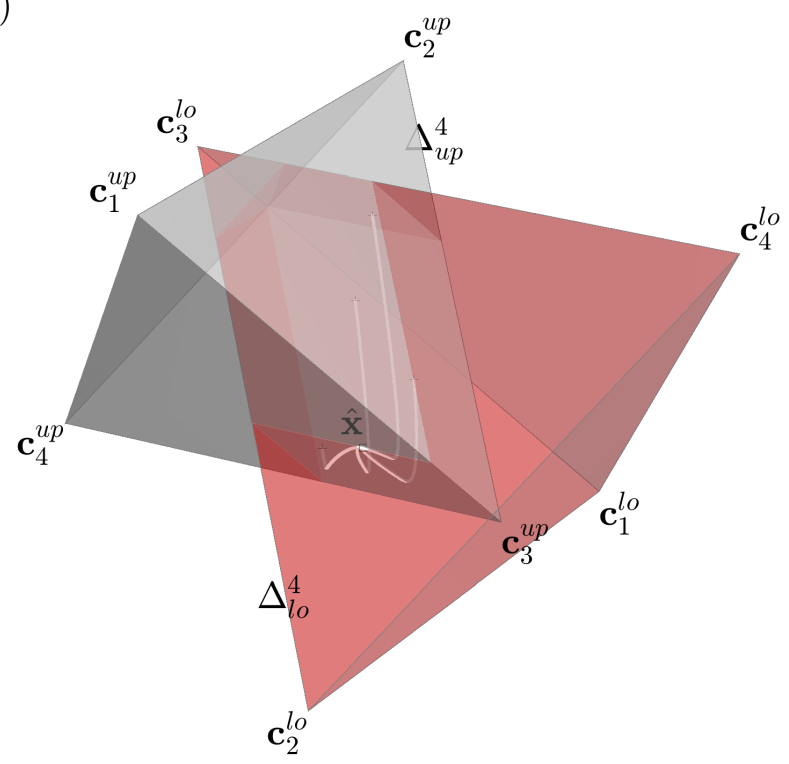

Fig. 8. An example of simplices $\Delta_{u p}^{4}$, and $\Delta_{l o}^{4}$, both in $\mathbb{R}^{4}$. Example of intersection subset defined by 9 vertices.

Although this example is useful to observe that IEED exhibits a certain computational advantage over MSD, the important drawback of MSD in the representation of hosting capacities lies in the problem of finding all the vertices of the intersection. This is specially problematic when the number of dimensions is increased, and when the constraints are not homogeneous for all the variables. In fact, the number of vertices of the intersection can be much larger than $K$ and enumerating all of them is a combinatorial problem, hard to solve computationally [18].

For instance, two examples of upper and lower constraints are presented in Figs. 7-8, both with four trajectories followed with the IEED and the corresponding escort functions. Fig. 7 shows the case where the feasible region is defined by the intersection between $\Delta_{l o}^{4}=\left\{x_{1} \geq\right.$ $0.1, x_{2} \geq 0, x_{3} \geq 0.2, x_{4} \geq 0.2, x_{1}+x_{2}+x_{3}+$ $\left.x_{4}=1\right\}$, and $\Delta_{u p}^{4}=\left\{x_{1} \leq 0.6, x_{2} \leq 0.3, x_{3} \leq\right.$ $\left.0.6, x_{4} \leq 0.5, x_{1}+x_{2}+x_{3}+x_{4}=1\right\}$. In this case, the intersection has 10 vertices and the feasible equilibrium state is $\hat{\mathbf{x}}=[0.1,0.3,0.275,0.325]^{\mathrm{T}}$. On the other hand, Fig. 8 shows the case where the feasible region is defined by the intersection between $\Delta_{l o}^{4}=\left\{x_{1} \geq 0.2, x_{2} \geq\right.$ $\left.0.1, x_{3} \geq 0, x_{4} \geq 0.1, x_{1}+x_{2}+x_{3}+x_{4}=1\right\}$, and $\Delta_{u p}^{4}=\left\{x_{1} \leq 0.3, x_{2} \leq 0.4, x_{3} \leq 0.5, x_{4} \leq\right.$ $\left.0.3, x_{1}+x_{2}+x_{3}+x_{4}=1\right\}$. In this final example, the intersection has 9 vertices and the feasible equilibrium state is $\hat{\mathbf{x}}=[0.2,0.4,0.175,0.225]^{\mathrm{T}}$. As it can be observed, the number of vertices of the intersection is not easily predictable and this also imposes a problem for the enumeration strategy [18]. 


\section{Conclusions}

In this paper, a detailed comparison through illustrative examples between MSD and IEED has been given. A main drawback of MSD in representing hosting capacities lies in the difficulty of finding all the vertices of the intersection. As it has been observed, IEED shows a certain computational advantage over MSD where the intersection is represented without knowledge of its vertices.

\section{REFERENCES}

[1] J. Hofbauer and K. Sigmund, "Evolutionary games and population dynamics." Cambridge University Press, 1998.

[2] N. Quijano, C. Ocampo-Martinez, J. Barreiro-Gomez, G. Obando, A. Pantoja, and E. Mojica-Nava, "The role of population games and evolutionary dynamics in distributed control systems: The advantages of evolutionary game theory," IEEE Control Systems, vol. 37, no. 1, pp. 70-97, 2017.

[3] J. Barreiro-Gomez, N. Quijano, and C. Ocampo-Martinez, "Constrained distributed optimization: A population dynamics approach," Automatica, vol. 69, pp. 101-116, 2016.

[4] H. Tembine, E. Altman, R. El-Azouzi, and Y. Hayel, "Evolutionary games in wireless networks," IEEE Transactions on Systems, Man, and Cybernetics, Part B (Cybernetics), vol. 40, no. 3, pp. 634-646, 2010.

[5] A. Ovalle, J. Fernandez, A. Hably, and S. Bacha, "An electric vehicle load management application of the mixed strategist dynamics and the maximum entropy principle," IEEE Transactions on Industrial Electronics, vol. 63, no. 5, pp. 30603071, 2016.

[6] A. Ovalle, A. Hably, S. Bacha, G. Ramos, and J. Hossain, "Escort evolutionary game dynamics approach for integral load management of electric vehicle fleets," IEEE Transactions on Industrial Electronics, vol. 64, no. 2, pp. 1358-1369, 2017.

[7] A. A. Khan, M. Abolhasan, and W. Ni, "An evolutionary game theoretic approach for stable and optimized clustering in vanets," IEEE Transactions on Vehicular Technology, vol. 67, no. 5, pp. 4501-4513, 2018.

[8] A. A. A. Abass, L. Xiao, N. B. Mandayam, and Z. Gajic, "Evolutionary game theoretic analysis of advanced persistent threats against cloud storage," IEEE Access, vol. 5, pp. 84828491, 2017.

[9] K. Coninx, G. Deconinck, and T. Holvoet, "Who gets my flex? an evolutionary game theory analysis of flexibility market dynamics," Applied Energy, vol. 218, pp. 104-113, 2018.

[10] M. Pelillo, "Replicator dynamics in combinatorial optimization," in Encyclopedia of Optimization, Second Edition, 2009, pp. 3279-3291.

[11] J. W. Weibull, Evolutionary game theory. MIT press, 1997.

[12] A. Menon, "Replicators \& complementarity: Solving the simplest complex system without simulation," in Computational Science - ICCS 2001, International Conference, San Francisco, CA, USA, May 28-30, 2001. Proceedings, Part II, 2001, pp. 922-934.

[13] D. E. Fryer, "On the existence of general equilibrium in finite games and general game dynamics," arXiv preprint arXiv:1201.2384, 2012.

[14] M. Harper and D. Fryer, "Incentive processes in finite populations," arXiv preprint arXiv:1306.2389, 2013.

[15] M. Harper, "Escort evolutionary game theory," Physica D: Nonlinear Phenomena, vol. 240, no. 18, pp. 1411 - 1415, 2011.
[16] A. Ovalle, J. Fernandez, A. Hably, and S. Bacha, "An electric vehicle load management application of the mixed strategist dynamics and the maximum entropy principle," IEEE Trans. Ind. Electron., vol. 63, no. 5, pp. 3060-3071, 52016.

[17] M. Harper and D. Fryer, "Lyapunov functions for time-scale dynamics on riemannian geometries of the simplex," Dynamic Games and Applications, vol. 5, no. 3, pp. 318-333, Sep 2015. [Online]. Available: https://doi.org/10.1007/s13235014-0124-0

[18] D. Avis and K. Fukuda, "Reverse search for enumeration," Discrete Applied Mathematics, vol. 65, no. 1-3, pp. 21 - 46, 1996, first International Colloquium on Graphs and Optimization. 